\title{
Probing interactions between DNA repair proteins and FUS in mammalian cells on microtubule bench
}

\author{
Mamontova E. ${ }^{1,2 *}$, Sukhanova M. ${ }^{1}$, Joshi V. ${ }^{2}$, Lavrik O. ${ }^{1}$, Pastré D. ${ }^{2}$ \\ ${ }^{1}$ Institute of Chemical Biology and Fundamental Medicine, SB RAS, Novosibirsk, Russia \\ ${ }^{2}$ SABNP, University of Evry, INSERM U1204, University Paris-Saclay, Evry, France \\ *evgeniya.mamontova@univ-evry.fr
}

Key words: DNA repair, PARP1, RNA-binding proteins, FUS, membraneless compartments

Motivation and Aim: The goal of this project is to investigate the role of RNA-binding protein FUS in DNA repair mechanisms. FUS (FUS/TLS, Fused in Sarcoma) is a member of FET family. It is one of the most enriched protein factors recruited in DNA damage regions and one of the most highly poly(ADP-ribosyl)ated nuclear RNA-binding proteins after oxidative stress. Moreover, FUS is known to form membraneless compartments through interactions occurring between its low complexity domains. It was previously demonstrated that FUS in the presence of poly(ADP-ribose) (PAR) generates dynamic compartments in which damaged DNA accumulates [1]. We hypothesize that the formation of such compartments at DNA damage sites may also help to concentrate DNA repair factors where they are needed the most. However, which DNA repair factors can be recruited to FUS-rich membraneless compartments is yet to be determined.

Methods and Algorithms: We utilized a new "microtubule bench" method to analyze the mixing/demixing of two different phases in a cellular context [2]. The principle is the following: HeLa cells are transfected by plasmids encoding the sequence of the protein of interest fused to Tau (a microtubule-associated protein) and to the sequence of GFP or RFP. Through their fusion to Tau the proteins are confined on microtubules and quantitative parameters defining their spatial segregation are measured along the microtubule network in cells.

Results: We obtained 10 mammalian expression vectors encoding the sequences of DNA repair proteins (PARP1, XRCC1, APE1, POL $\beta$, Lig1, Lig3, TOP1, PP1 $\alpha$, PSPC1 and NONO) fused to the sequences of RFP and Tau proteins. Then we performed a cotransfection of HeLa cells with both FUS-GFP-Tau vector and RFP-Tau vector containing the sequence of DNA repair protein, fixed the cells and prepared the samples for fluorescence microscopy. We observed that two DNA repair proteins PARP1 and APE1 are mixed with FUS on the microtubules.

Conclusion: The results suggest that PARP1 and APE1 can be brought to FUS-rich membraneless compartments. It means that PARP1 can interact with FUS directly or via interaction of FUS with PAR attached to PARP1. The mixing between APE1 and FUS probably originates from the interaction of both proteins with PAR. Acknowledgements: The study is supported by RSF (project No. 20-14-00086).

\section{References}

1. Singatulina A.S. et al. PARP-1 activation directs FUS to DNA damage sites to form PARGreversible compartments enriched in damaged DNA. Cell Reports. 2019;27(6):1809-1821.

2. Maucuer A. et al. Microtubules as platforms for probing liquid-liquid phase separation in cells application to RNA-binding proteins. J. Cell Sci. 2018;131(11). 